\title{
Engineering, Concepts and Video Clips
}

\author{
http://dx.doi.org/10.3991/ijep.v3iS1.2413 \\ J.C. Marques ${ }^{1}$, J. Quintela ${ }^{2}$, M.T. Restivo ${ }^{1}$ and V.M Trigo ${ }^{3}$ \\ ${ }^{1}$ Universidade do Porto, Porto, Portugal \\ ${ }^{2}$ PT Inovação, Aveiro, Portugal \\ ${ }^{3}$ Instituto Politécnico de Lisboa, Lisboa, Portugal
}

\begin{abstract}
Nowadays there is a huge diversity of multimedia tools available for helping to make concepts more easily and objectively understood. Such tools may be instrumental in science and technology for establishing very suggestive connections between theoretical models and the corresponding real life phenomena. An effective use of these tools in the form of short video clips linking experimental laboratory evidence with natural events is illustrated in this work.
\end{abstract}

Index Terms-Engineering concepts, instructional aids, multimedia communication, videos.

\section{INTRODUCTION}

The effectiveness of communication can be directly related to the medium of transmission. Video excels as an invaluable medium in the knowledge acquisition process in the context of Higher Education, besides being instrumental to communicate science and to disseminate Research, Development and Innovation (R\&D\&I) activities [1]. Video is a dynamic, multi-sensorial medium with an outstanding capacity to attract the viewer's attention and to convey complex messages. Nevertheless it is essential to always take into account the objectives of the message and the needs and expectations of the target audience.

This work intends to show how video may be explored in the form of small clips in the context of instructional processes in Civil Engineering. The aim of this type of video clip is the concise transmission of an objective, engaging the viewer's attention and arousing interest in the subject by linking experimental evidence of concepts with real life events.

\section{The Role OF Higher EducAtion InSTITUTIONS}

Higher Education Institutions (HEI) need to attract students and to keep them interested in their courses. But it has been recurrently detected that some students have difficulty acquiring knowledge, particularly when it has to be associated to practice (i.e. "put to work"). Perhaps things did not use to be like this but in these times of change the process of knowledge formation is also changing. Linear models are being replaced by non-linear and interactive ones.

Students "need to visualize experiments, facts and observations of daily life in order to be able to associate them to the theories ministered in the classroom" [2].

Given the tremendous scientific and technological evolution of today's world the teaching/learning process must substantiate, more than ever before, the correlation between concepts and reality.

Teachers should not deliver contents without associating them to the world around us and to concepts or practices previously acquired. Knowledge is too often transmitted and supported in memorization, lacking interaction and context. This approach, allied to the increase in the number of young people engaged in tertiary education, has proved to be quite inefficient in providing students with understanding.

This represents a deep change in traditional pedagogy, which means that a considerable amount of complexity is being added to the teaching/learning process. A transdisciplinary approach is needed to deal with this situation.

Furthermore, HEIs also need to adapt their message to their public, not only to educate students efficiently but also to achieve a broad dissemination of the HEIs work, both within the academic community, for potential students and for the public at large. This type of diffusion may employ a wide range of communication tools within which video stands out due to its ability for engaging the viewer's attention and for arousing interest in the subject.

Thus, teachers and researchers must develop new competencies in the communication field towards what we could call hybrid competencies, with video handling and producing being among them. These reflections link to recent studies about science of learning and education [3].

\section{VIDEO}

Humans trust their visual perception of the world to convey ideas, feelings and knowledge. In particular video excels as an aid for the assimilation of educational content and as an effective tool for the dissemination of technoscience. It has the power to convey complex scientific ideas: images in motion, combined with colour and sound, easily attract the viewer's attention and help to clarify scientific concepts. Moreover the video camera has the priceless capacity to capture unique moments, unrepeatable in space or in time [2].

Indeed, the use of video clips in engineering education brings an extra dimension at an affordable cost. Furthermore video clips can be used beyond the traditional classroom, being a useful tool in e-learning, blearning and distance-learning environments, which are spreading within HEI. They should be seen as learning objects, and "like LEGO bricks, learning objects can be arranged in different ways to build different learning experiences" [4]. 


\section{SPECIAL FOCUS PAPERS \\ ENGINEERING, CONCEPTS AND VIDEO CLIPS}

Access to video production tools has become absolutely trivial, nowadays. A short video clip for educational purposes can be easily produced with a smart phone, a personal computer and free software. Moreover, students can be easily engaged in the very process, with substantial gains in motivation being achieved [5].

However, the use of video for dissemination of technoscience is sometimes criticized with the argument that images may be ambiguous and misleading.

Some authors from the psychological field have discussed the constraints linked to multimedia learning. They alert to the risks of an overuse of elements originating a redundancy effect that could harm the message effectiveness [6]. This is one good reason for teachers and researchers to acquire the above mentioned hybrid competencies.

There is still work to be done in this area, mostly due to the lack of rules. But in spite of their absence one may always resort to the good principles and avoid the bad examples. And, again, engage students in the evaluation process.

\section{Video As An EngINEERING TEACHING TOOL}

YouTube, the popular video sharing website, has 72 hours of video uploaded every minute, over 800 million unique visitors per month and more than 1 trillion views in 2011 [7].

The use in a Soil Mechanics lecture of two videos taken from YouTube showing people walking on (and sinking into) quicksand $[8,9]$ led to a lively discussion with positive results in terms of student engagement and knowledge acquisition. This attests to the importance of visualization, as well as to the paramount role that video may play as an instructional aid.

Physical phenomena may be described in a mathematical, abstract manner, based on their governing differential equations. From an educational point of view it is essential to provide whenever possible a suggestive representation to facilitate the acquisition of concepts through the visualization of theory in action [10].

The flow of water through soil, the so called seepage or groundwater flow, is one of the subjects taught to Civil Engineering students in Soil Mechanics courses. Year after year students have shown some trouble in understanding basic concepts, mostly because of their lack of feeling for the underlying physics. This may be attributed to the fact that Civil Engineering curricula tend to develop and train more extensively the student sensitivity for mechanical and structural problems, with less emphasis on those involving soil-water interaction.

In order to help improve the student perception of the physics involved in groundwater flow an experimental didactic apparatus for small scale studies has been designed and developed with the aim of providing a clear visualization of the phenomena involved [11,12]. An IP camera provides a video link between the laboratory and the lecture theatre (Fig. 1). The equipment has been used to perform several practical experiments whose objective is to make students aware of the qualitative and quantitative aspects of the physics of flow through porous media, with emphasis on situations relevant for Civil Engineering, such as the flow into a cofferdam, under a curtain or through an earth dam (Fig. 2). In this last case this has involved the preparation of small scale models of several typical earth dam cross-sections equipped with seepage control devices frequently used in such structures, namely a drainage blanket, a toe drain, a chimney drain and a central core with low permeability [13].

The experiments include the visualization of flow lines and the construction of flow nets, the measurement of hydraulic head loss and the verification of Darcy's law, the demonstration of hydraulic instability phenomena, among other features.

The preparation of each of the various small scale setups that are provided by the equipment flexibility takes at least one full day of hard work. Given the equipment generous internal dimensions $(200 \mathrm{~cm}$ long, $58.5 \mathrm{~cm}$ high and $20 \mathrm{~cm}$ wide) well over $200 \mathrm{~kg}$ of sand have to be taken out and shaped back in into a new configuration that may involve some complexity due to the presence of various soil types and/or seepage control devices.

This is precisely where video recording is most helpful. Students may have direct contact to only one particular small scale model but they have access to video clips of their whole range of configurations which are not feasible to reproduce in a short time. Each configuration is thoroughly documented in a video recording and this is an extremely convenient complement.

Although the emphasis is on the issues that contribute to a safe design whenever groundwater flow is involved, it is also possible to observe and reproduce in the equipment the type of undesirable phenomena that have led to catastrophic disasters in some unfortunate situations, with loss of lives and extensive property damage.

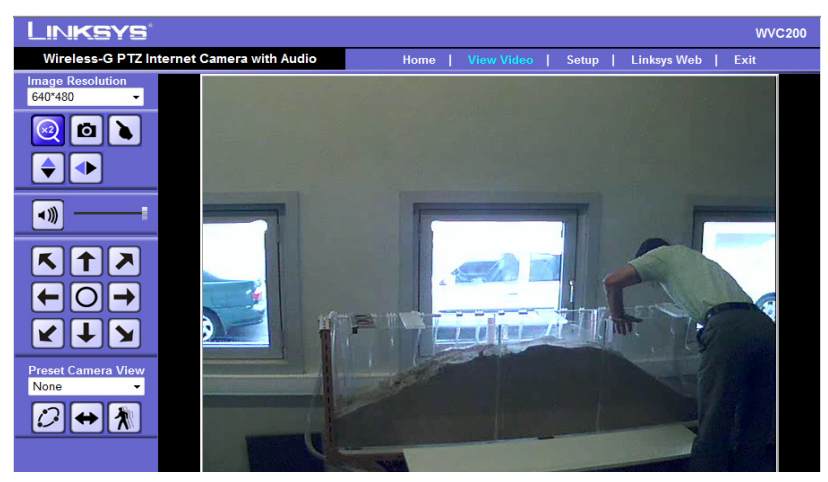

Figure 1. Lecturing through the intranet

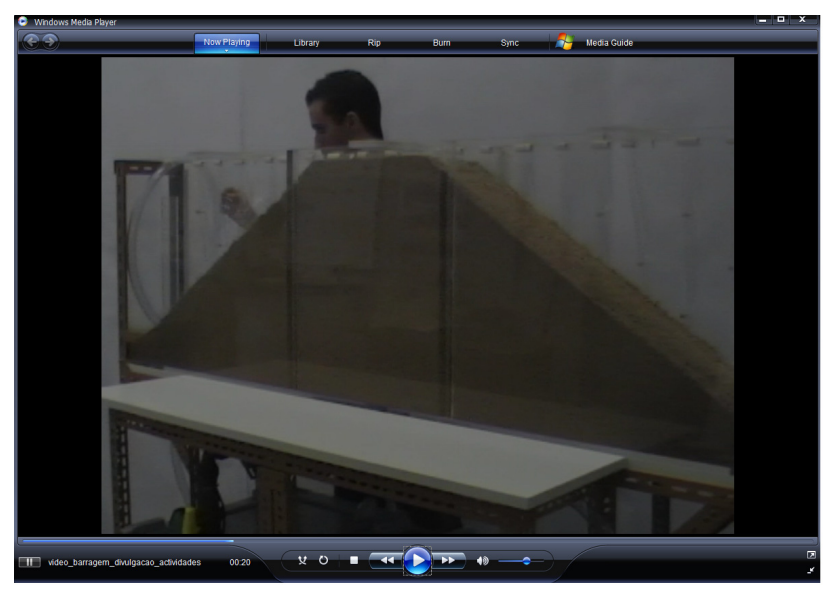

Figure 2. Earth dam small scale model 


\section{SPECIAL FOCUS PAPERS \\ ENGINEERING, CONCEPTS AND VIDEO CLIPS}

One of them is the erosion of the downstream slope of a homogeneous dam without internal drain due to the emergence of seepage flow on the dam face. This process will eventually cause the dam failure. Another dramatic event is the overtopping of an earth dam. The flow of water over the crest of the dam has disastrous consequences for its structural integrity.

Video clips are an excellent medium to relate in an educational manner small scale simulations of this kind with footage of real events.

\section{CASE StUdy}

A video clip entitled "Earth Dam Disasters" has been prepared and included in an online survey using Qualtrics software. The English version of the survey is available at https://feup.eu.qualtrics.com/SE/?SID=SV_ekOC1KGoS

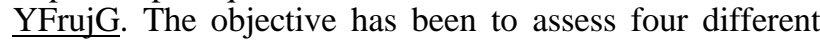
aspects of the video clip as an educational tool. The target group has included 4th year students of the Integrated Master in Civil Engineering as well as a group of selected academics of this area.

\section{A. Introductory Text}

The survey starts with a small introductory text transcribed below.

"The designation large dam is attributed to those whose height exceeds 15 metres. Over $70 \%$ of large dams are embankment dams (earth or rockfill dams) according to the World Register of ICOLD (International Commission on Large Dams). The 335 metre high Rogun Dam, under construction in Tajikistan, will be the world highest embankment dam when completed.

Embankment dams have safety devices and monitoring equipment because the collapse of this type of structure would cause large losses in human and property terms. Small scale models of embankment dams highlight various aspects of their behaviour while also enabling the simulation of extreme scenarios, such as overtopping.

In complement to the experimental model a short video clip (not exceeding 2 minutes) has been prepared in order to stimulate the curiosity and to illustrate the relevance of the topic in a quick and suggestive manner."

\section{B. Questionnaire}

The introductory text is followed by a small questionnaire that starts by establishing the age and gender profile of the sample. Then four questions about the video clip are formulated.

1. How do you rate the small scale study equipment used in the video clip in terms of potential as a teaching tool?

2. How do you rank the value of the video clip for the exploration of features of groundwater flow (e.g., flow lines)?

3. How do you grade the effectiveness of the video clip as a vehicle for dissemination of the topic "Earth dam disasters"?

4. How do you rate the impact of the contrast established between the model simulated disaster and the real catastrophic event documented in the video clip?

The 4-option response scale includes Useless/Not very useful/Useful/Very useful.

\section{Results}

In total 86 replies have been received (74\% from males). The sample is mostly composed by young people (61\% are in the 15 to 34 age group) (Fig. 3).

Figs. 4 to 7 illustrate the results of the four questions.

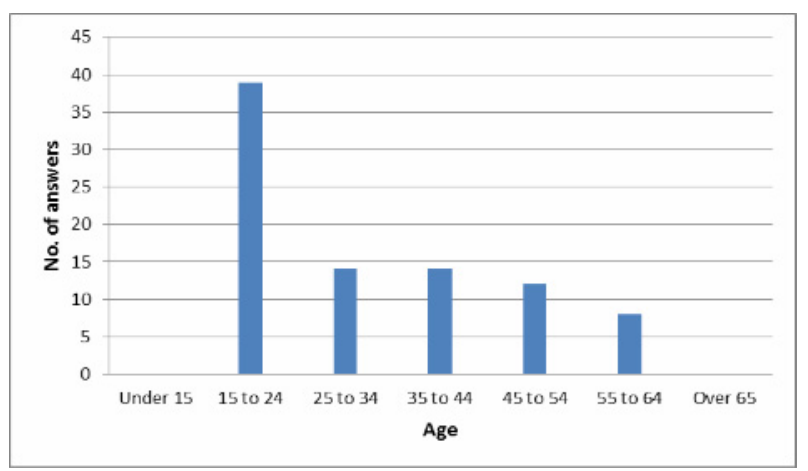

Figure 3. Age distribution of the sample

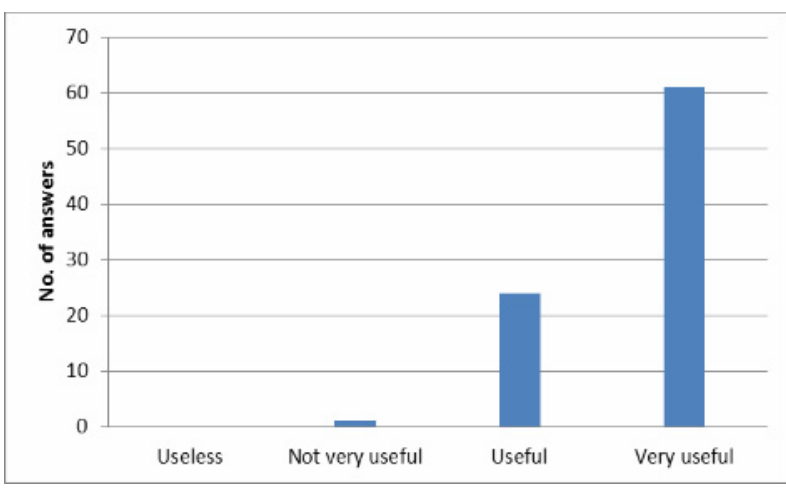

Figure 4. Results of Question 1

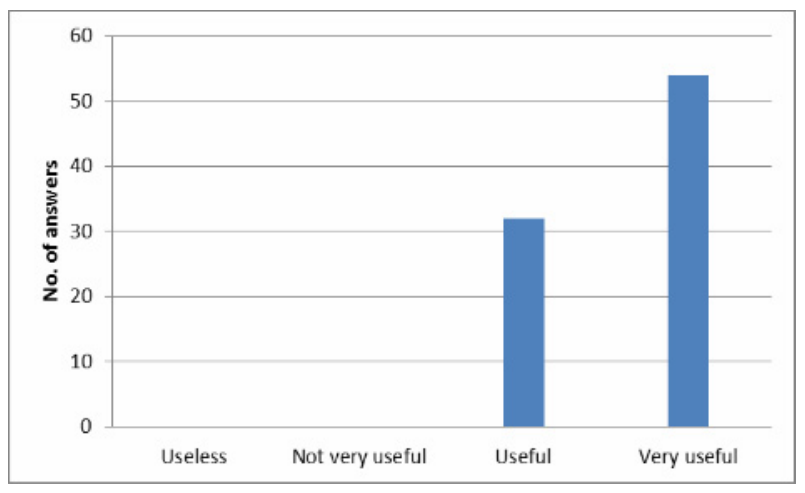

Figure 5. Results of Question 2

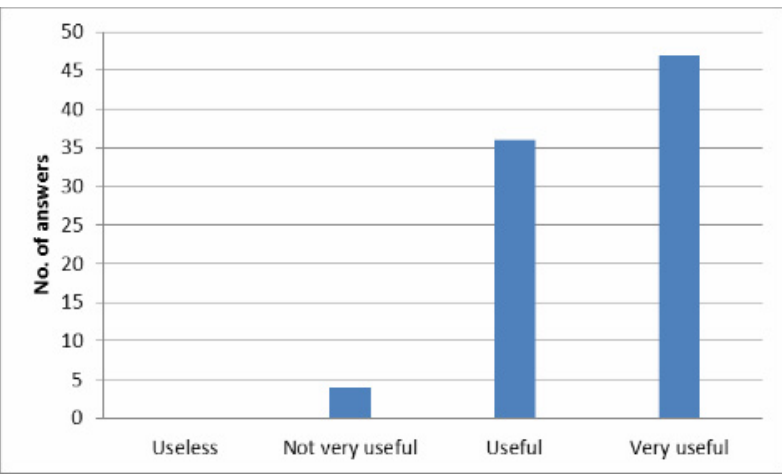

Figure 6. Results of Question 3 


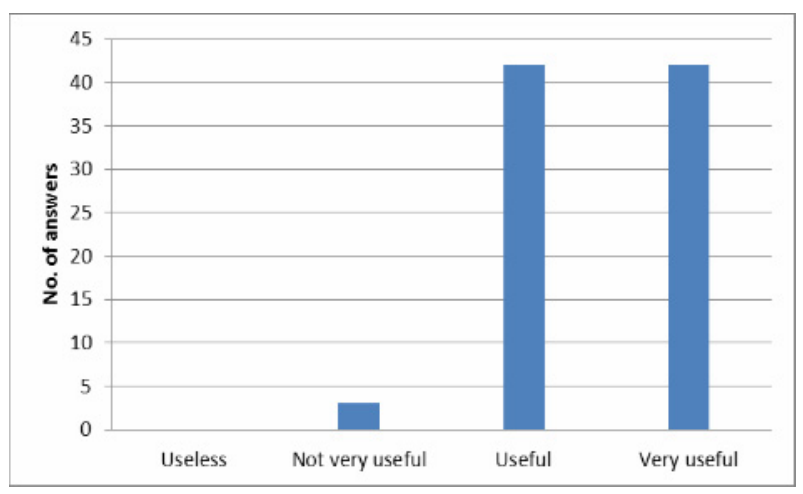

Figure 7. Results of Question 4

\section{Analysis}

The teaching tool potential of the small scale laboratory equipment for groundwater flow studies is assessed as very positive $(70.9 \%)$.

This also applies to the perception of the value of the video clip for exploring features of groundwater flow phenomena, which is considered a very valid contribution (62.8\%) for understanding the subject.

The video clip is judged an effective (41.9\%) or very effective (54.7\%) vehicle for dissemination of the topic "Earth Dam Disasters".

Finally the contrast established in the video clip between small scale disasters and real extreme events is rated as having substantial or very substantial impact (48.8\% in both cases). This preliminary study shows that video clips are an excellent medium for the transmission of messages in an academic context by illustrating the correlation between theory and reality.

\section{E. Discussion}

The survey had the objective of assessing four complementary dimensions of a short video clip on a specific geotechnical subject.

The feedback obtained shows in a consistent and encouraging manner that the overall rating of the video clip by the target group has been very good.

If an image is indeed worth one thousand words as the saying goes, then video clips can be very powerful and dynamic teaching aids, with the capacity to convey information in a concise and suggestive way.

Video clips can therefore provide a very substantial added value by helping to establish visual connections between concepts and applications in the context of Engineering Education.

\section{FINAL REMARKS}

In addition to being extremely popular among the young generations, video is a very effective medium particularly for the dissemination of methods, procedures and results in science and technology fields. It requires less space and provides more objectivity than the verbal form for the same amount of information [14]. The potential of video clips in Engineering Education has been already perceived [15] and is being increasingly explored to stimulate curiosity, attract the attention and motivate students to deepen their knowledge.

\section{REFERENCES}

[1] J. V. Quintela, The Use of Video for the Divulgation of R\&D\&I activities, MSc Thesis in Multimedia (in Portuguese), FEUP, Porto, 2011.

[2] M. Busse, "Online video is transforming perceptions of science," New Scientist Tech, no. 2612, 11 July 2007.

[3] T. Jörg, B. Davis and G. Nickmans, "Towards a new, complexity science of learning and education", Educational Research Review, 2, pp. 145-156, 2007. http://igitur-archive.library.uu.nl/ivlos/20080207-200602/j_246_rg-towards\%20a\%20new\%20complexity\%20 science.pdf. http://dx.doi.org/10.1016/j.edurev.2007.09.002

[4] P. Case and J. Hino, "A powerful teaching tool: self-produced videos,” Journal of Extension, vol.48 (1), February 2010. http://www.joe.org/joe/2010february/pdf/JOE_v48_1tt3.pdf.

[5] E. Bravo, B. Amante, P. Simo, M. Enache, V. Fernandez, "Video as a new teaching tool to increase student motivation," 2011. http://upcommons.upc.edu/e-prints/bitstream/2117/12717/1/bravoamante.pdf.

[6] R. E. Mayer, J. Heiser and S. Lonn, "Cognitive constraints on multimedia learning: when presenting more material results in less understanding,” Journal of Educational Psychology, vol. 93(1), pp. 187-198, March 2001. http://visuallearningresearch.wiki.educ. msu.edu/file/view/Mayer, + Heiser, $+\% 26+$ Lonn+\%282001\%29.pdf http://dx.doi.org/10.1016/j.edurev.2007.09.002

[7] http://www.youtube.com/t/press_statistics

[8] http://www.youtube.com/watch?v=omBFjFGwRhs

[9] http://www.youtube.com/watch?v=b5Hr5a1n7uY\&feature=relmfu

[10] M. T. Restivo and J. C. Marques, "Art and multimedia in science and technology,” WCCA 2010 - World Congress on Communication and Arts, Guimarães.

[11] J. C. Marques and C. R. Ferreira, "A project based learning case study - Development of a didactic equipment for groundwater flow problems," Proc. PAEE 2009, $1^{\text {st }}$ Ibero-American Symposium on Project Approaches in Engineering Education, pp. 247-253, Dinis Carvalho et al. (eds.), Guimarães.

[12] C. R. Ferreira, Development of a Laboratory Equipment for Small Scale Study of Seepage Problems, Master Thesis in Civil Engineering (in Portuguese), FEUP, Porto, 2008.

[13] M. S. P. Unas, Experimental and Numerical Study of Groundwater Flow in Small Scale Model, Master Thesis in Civil Engineering (in Portuguese), FEUP, Porto, 2010.

[14] M. Pasquali, "Protocol videos: the implications for research and society,” EMBO Reports, 8 (8), 712-716, 2007. http://dx.doi.org/ 10.1038/sj.embor.7401037

[15] P. Bell, B. Lewenstein, A.W. Shouse and M.A. Feder, Learning Science in Informal Environments: People, Places, and Pursuits. Washington: The National Academies Press, 2009.

\section{AUTHORS}

J. C. Marques is with Universidade do Porto, Faculdade de Engenharia, Porto, Portugal (e-mail: jmarques@fe.up.pt).

J. Quintela is with PT Inovação, Aveiro, Portugal (email: joanaqui@gmail.com).

M. T. Restivo is with Universidade do Porto, Faculdade de Engenharia and with UISPA Research Unit at IDMEC-Pólo FEUP, Porto, Portugal (e-mail: trestivo@fe.up.pt).

V. M. Trigo is with Escola Superior de Comunicação Social, Instituto Politécnico de Lisboa, Lisboa, Portugal (e-mail: vtrigo@escs.ipl.pt).

This article is an extended and modified version of a paper presented at the IGIP2012 conference, held 26 - 28 September 2012, in Villach, Austria. Received 1 December 2012. Published as resubmitted by the authors 28 January 2013 .. 